\title{
Are Combined Techniques Using Video Laryngoscopes and Dynamic Stylets Superior to Fiberoptic Techniques for Anticipated Difficult Intubations? A Retrospective Case Control Study
}

\author{
Ashka Shah \\ University of Utah Health Care \\ Lauren Knecht \\ University of Utah Health Care \\ Cameron Jacobson \\ University of Utah Health Care \\ Sean Runnels ( $\nabla$ sean.runnels@hsc.utah.edu ) \\ University of Utah Health Care

\section{Angela Presson} \\ University of Utah Health Care \\ Chong Zhang \\ University of Utah Health Care

\section{Kathryn Durnford} \\ University of Utah Health Care
}

\section{Research Article}

Keywords: Difficult intubation, Combined technique, First pass success, Intubation time, Intubation trauma, Articulating introducer, Dynamic stylets

Posted Date: May 5th, 2021

DOI: https://doi.org/10.21203/rs.3.rs-446796/v1

License: (c) (i) This work is licensed under a Creative Commons Attribution 4.0 International License. Read Full License 


\section{Abstract}

Difficult intubations can require advanced intubation techniques. Studies point to potential advantages of combined techniques using video laryngoscopes $(\mathrm{VL})$ and dynamic stylets for anticipated difficult intubations. This study is designed to compare combined techniques to awake and asleep fiberoptic (FOB) techniques.

Methods: 138,387 consecutive anaesthesia cases were reviewed for use of: FOB awake, FOB asleep, or combined technique (VL for visualization and either a FOB or a novel $\mathrm{TCl}^{\mathrm{TM}}$ articulating introducer ((TCl ${ }^{\mathrm{TM}}$; Through The Cords, LLC; Salt Lake City, UT)) as dynamic stylets as a primary approach for anticipated difficult intubations. Primary end points measured: first attempt success rate, failure to intubate with the primary technique, "in-room to intubation" time, reported traumatic intubation rate, and reported ease of intubation.

Results: Significant differences were found between techniques. First pass success rate was highest in combined techniques (either VL + FOB or VL + $\mathrm{TCl}^{\mathrm{TM}}$ ) (88.7\%) followed by FOB awake (74.2\%, P<0.001) and FOB asleep (80.7\%, $\mathrm{P}=0.06)$. "Failure to intubate with the primary technique" was lowest in combined techniques (1.8\%) followed by FOB asleep $(4.6 \%, \mathrm{P}=0.11)$ and FOB awake $(9.2 \%, \mathrm{P}=0.002)$. "In room to intubated" time was fastest in combined techniques (13.0 minutes) followed by FOB asleep (15.1 minutes, $\mathrm{P}=0.002)$ and $\mathrm{FOB}$ awake $(21.2$ minutes, $\mathrm{P}<0.001)$. Combined techniques were rated as 'easy' more often (72\%) followed by FOB asleep (62.9\%, P=0.12) and FOB awake $(38.2 \%$, $\mathrm{P}<0.001)$. Combined techniques were rated as "atraumatic" more often $(91.1 \%)$ followed by FOB asleep $(89.4 \%, P=0.91)$ and FOB awake $(75.8 \%, P<0.001)$.

In subgroup analysis of combined techniques, $\mathrm{VL}+\mathrm{TCl}^{\mathrm{TM}}$ had the highest first attempt success rate (90.2\%), lowest failure rate $(1 \%, P=0.56)$, and shortest "in room to intubated time" (12.1 minutes, $P=0.12)$. It was also rated as "easy" (83.3\%, $\mathrm{P}<0.001)$, and "atraumatic" $(96.1 \%, \mathrm{P}=0.009)$ more often than $\mathrm{VL}+\mathrm{FOB}, \mathrm{FOB}$ awake or FOB asleep.

Conclusions: Combined techniques outperformed FOB techniques in terms of effectiveness, speed, ease of use, and patient injury in patients with risk factors for difficult intubation. As a sub-group of combined technique, $\mathrm{VL}+\mathrm{TCl} \mathrm{TM}^{\mathrm{TM}}$ outperformed all other techniques. Combined techniques should be considered when managing difficult intubations.

\section{Background}

Difficult intubation remains a significant problem. There is clear correlation between number of intubation attempts, major complications and mortality. ${ }^{12}$ In the event of an expected or unexpected difficult intubation with direct laryngoscopy (DL) and/or VL, it is important to have alternative advanced airway techniques readily available as a primary or rescue approach to intubation. Common advanced intubation techniques include awake FOB, asleep FOB, and combined techniques using $\mathrm{VL}$ with dynamic stylets such as $\mathrm{VL}+\mathrm{FOB}$ or $\mathrm{VL}+\mathrm{TCl}^{\mathrm{m}}$. ${ }^{3}$ When managing difficult intubations, the optimal technique should be readily available, fast, effective, easy to use, and atraumatic.

Intubations can be difficult or fail for two main reasons: 1) difficult glottic visualization and/or 2) difficult tracheal access. The natural airway is formed by two directionally opposing curves, the oro-pharyngeal curve and the pharyngoglotto-tracheal curve, with the inflection point at the glottis. These curves essentially form a serpentine pathway from the lips to the mid trachea. When DL is used, these curves are flattened allowing direct visualization of the glottis and "lineof-sight" tracheal access to take place. VL consistently improves glottic visualization compared to DL by allowing indirect visualization of the glottis around the oro-pharyngeal curve without the need to flatten either curve. However, this unchanged serpentine pathway from the upper airway into the mid trachea can make tracheal access difficult or even impossible with VL. ${ }^{4}$ Difficult tracheal access has been demonstrated to occur in up to $11.4 \%$ of VL uses. ${ }^{6}$ Failure to 
navigate into the trachea accounted for $76 \%$ intubation failures in a study of six different intubations using VL. ${ }^{7}$ This is the paradox of VL; superior visualization comes at the cost of sometimes challenging tracheal access.

VL was introduced into practice almost two decades ago intent on solving visualization problems associated with DL. There has been little progress in tracheal access technology to compliment VL. Commonly available bougies and malleable stylets were designed for DL. Pre-curved rigid stylets designed for VL compensate for the oro-pharyngeal curve only. Once at the glottis, the fixed curve cannot be reshaped to follow the directionally opposed pharyngo-glotto-tracheal curve for precise navigation into the trachea. A few introducers have been specifically designed to dynamically navigate the serpentine pathway associated with VL. ${ }^{89}$ As of yet, none have achieved widespread use.

A combined technique, using VL for visualization paired with a dynamic stylet for tracheal access, aims to take advantage of the superior visualization provided by $\mathrm{VL}$ and the active navigational abilities provided by dynamic stylets. Key features of dynamic stylets are an articulating tip for precision navigation and a flexible shaft that can conform to the entire serpentine pathway of the airway.

Recent literature suggests that combined techniques may have advantages over VL with a rigid stylet or FOBs alone when used to manage difficult intubations in terms of improved effectiveness and lower airway injury rates. 101112 Lenhart et al. demonstrated the ability of VL + FOB to rescue after failed VL with the standard rigid, pre-curved stylet. ${ }^{13}$ They also reported $20 / 20$ patients with cervical spine pathology and limited neck range of motion were successfully intubated using VL + FOB. However, it is not clear in this study whether the FOB was used for visualization or only for its navigational capabilities.

Sgalambro et al. studied a combined technique utilizing DL + FOB in unexpected difficult airways. All 253 patients with unexpected difficult intubations were successfully intubated using DL + FOB. ${ }^{14}$ The reported learning curve for DL + FOB was only 10 intubations.

Mazzinari et al. investigated the use of VL + FOB in which the FOB was used simply as a dynamic stylet in predicted difficult intubations. This study found that a dynamically shapable stylet resulted in significantly higher first pass rate, lower injury rate, and shorter intubation time when compared to the standard rigid pre-curved stylet. ${ }^{15}$

Shah et. al. conducted a retrospective chart review which demonstrated a $94 \%$ first attempt rescue rate and an overall success rate of $97 \%$ using a combined technique of $\mathrm{VL}+\mathrm{TCl}^{\mathrm{TM}}$ after failed laryngoscopy (with standard $\mathrm{VL}$ or $\mathrm{DL}$ ). ${ }^{11}$

Up to now, combined techniques have been studied in isolation as case reports, case series, or in controlled trials against $\mathrm{VL}$ with standard malleable or rigid stylets. Little is known about the performance of combined techniques in a real world setting in comparison to other common advanced airway techniques.

We designed a retrospective observational study to better understand the performance of combined techniques (either $\mathrm{VL}+\mathrm{FOB}$ or $\mathrm{VL}+\mathrm{TCl}^{\mathrm{TM}}$ ) against $\mathrm{FOB}$ awake and $\mathrm{FOB}$ asleep in the real world setting of a single large academic institution using a case controlled, observational, retrospective design.

\section{Methods}

This study was approved by the University of Utah Institutional Review Board \#0096411

\section{Study Design:}

Retrospective, observational, case controlled, electronic medical record review.

\section{Electronic Medical Record Review:}


138,387 consecutive cases from 5/12/15 through 8/20/2020 were analysed for those in which an advanced intubation technique was used as the primary approach for intubation. The inclusion criteria for advanced airway techniques were; asleep FOB awake, FOB asleep, $\mathrm{VL}+\mathrm{FOB}$, and $\mathrm{VL}+\mathrm{TCl}^{\mathrm{Tm}}$.

1,791 cases were identified in which an advanced airway technique was used.

These cases were sorted for any patient with at least one risk factor for difficult intubation. The inclusion criteria for an anticipated difficult airway were: Mallampati score of > 3; limited neck mobility; small mouth opening; body mass index $(\mathrm{BMI})>35$; neck circumference $>35.5 \mathrm{~cm}$ in males or $32 \mathrm{~cm}$ in females; and history of difficult intubation.

A total of 849 cases were identified in which an advanced technique was used as a primary approach to intubation in patients with at least one risk factor for difficult intubation. Analysed data for each case included: intubation devices used, free text airway procedure notes, case date, number of intubation attempts, "in room to intubated time" user rated ease of intubation, and user rated intubation trauma. See Fig. 1. for data flow and advanced intubation technique taxonomy.

These cases were then evaluated for these primary endpoints:

1) First attempt intubation success rate with primary advanced technique.

2) Failure to intubate using the primary advanced technique after 2 attempts.

3) Time from patient entry into the room to successful intubation ("in room to intubated” time).

4) User rated ease of intubation: easy, difficult.

5) User rated traumatic intubation: atraumatic, semi-traumatic, or traumatic.

\section{Rational for the choice of endpoints measuring effectiveness:}

First intubation attempt success- Optimizing first attempt intubation success is an important principle when planning any intubation. Understanding the first attempt success rates for different advanced intubation techniques can help clinicians when planning for intubations.

Failure of primary technique- We chose failure as three or more intubation attempts as an endpoint for our study as persistence with a failing technique is a risk factor for poor outcomes. ${ }^{16}$ After three or more attempts, an alternative technique should be considered. As there is no guarantee the alternative technique will be successful, limiting the number of attempts with the primary technique seems prudent.

'In room to intubation' time- Operating room time is a valuable and scarce resource. Aside from patient characteristics, the choice of intubation technique is known to affect the time needed to ready a patient before a surgical procedure can begin. ${ }^{17}$

Ease of use-Difficult intubations can be stressful for providers. Increased mental task load can reduce performance and increased risk during intubations. ${ }^{18}$ Airway techniques requiring advanced skills that are difficult to master and maintain may be avoided by practitioners even when their use is appropriate. ${ }^{19}$

\section{Groups and subgroups compared:}

Combined techniques as a group $\left(\mathrm{VL}+\mathrm{FOB}\right.$ and $\left.\mathrm{VL}+\mathrm{TCl}^{\mathrm{TM}}\right)$ was first compared to FOB awake and FOB asleep. $\mathrm{A}$ subgroup analysis of combined techniques was then carried out. VL + FOB as a subgroup was compared to FOB awake 
and FOB asleep, and $\mathrm{VL}+\mathrm{TCl}^{\mathrm{TM}}$ was compared to $\mathrm{VL}+\mathrm{FOB}, \mathrm{FOB}$ awake and FOB asleep. See Fig. 2 for comparative analysis flow.

\section{Statistical Analysis}

Patient demographics and outcomes were summarized descriptively and stratified by airway techniques. Continuous variables were summarized as mean and standard deviation (SD), median and inter quartile range (IQR) and range. Categorical variables were summarized as frequency and percent. For each variable, we compared: combined techniques as a group ( $\mathrm{VL}+\mathrm{FOB}$ and $\left.\mathrm{VL}+\mathrm{TCl}^{\mathrm{TM}}\right)$ to $\mathrm{FOB}$ asleep and FOB awake; $\mathrm{VL}+\mathrm{FOB}$ to $\mathrm{FOB}$ asleep and FOB awake; $\mathrm{VL}+\mathrm{TCl}^{\mathrm{TM}}$ to $\mathrm{VL}+\mathrm{FOB}, \mathrm{FOB}$ asleep and FOB awake using Wilcoxon rank sum test for continuous variables, and Chi-squared or Fisher's exact tests for categorical variables. Because we are making 10 pairwise comparisons for each variable, the significance of each comparison was assessed at $p=0.005$ to keep the family-wise type 1 error within 0.05 .

Time from "in room to intubation" was summarized descriptively across number of attempts, and the JonckheereTerpstra test was used to assess for a trend across number of attempts. For this comparison, significance was assessed at $p=0.05$. Density plots were created to graphically present the distribution of time from in room to intubation across airway techniques as well as number of attempts.

All analyses were conducted in R v4.0.x [R Core Team (2020). R: A language and environment for statistical computing. R Foundation for Statistical Computing, Vienna, Austria. URL https://www.R-project.org/], and all tests were two sided.

\section{Results}

The cohort included 934 intubation procedures and 849 patients. All filtering criteria were applied to the data already. Patient characteristics in the groups compared are presented in Tables 1a, 1b and 1c.

\begin{tabular}{|c|c|c|c|c|c|}
\hline Variable & $\begin{array}{l}\text { Combined }(\mathrm{N}= \\
168)\end{array}$ & $\begin{array}{l}\text { FOS asleep }(\mathrm{N}= \\
367)\end{array}$ & P-value & $\begin{array}{l}\text { FOS awake }(\mathrm{N}= \\
314)\end{array}$ & P-value \\
\hline $\mathrm{BMI} \geq 35$ & $41(27 \%)$ & $46(13.5 \%)$ & $\begin{array}{l}< \\
0.001 * c\end{array}$ & $84(27.8 \%)$ & $0.85^{c}$ \\
\hline Small mouth opening & $11(6.5 \%)$ & $20(5.4 \%)$ & $0.61^{c}$ & $24(7.6 \%)$ & $0.66^{c}$ \\
\hline Limited neck mobility & $55(32.7 \%)$ & $144(39.2 \%)$ & $0.15^{c}$ & $183(58.3 \%)$ & $\begin{array}{l}< \\
0.001 * \mathrm{C}\end{array}$ \\
\hline $\begin{array}{l}\text { Large neck } \\
\text { circumference }\end{array}$ & $10(6 \%)$ & $11(3 \%)$ & $0.10^{c}$ & $9(2.9 \%)$ & $0.10^{c}$ \\
\hline $\begin{array}{l}\text { Cervical spine } \\
\text { precautions }\end{array}$ & $8(4.8 \%)$ & $47(12.8 \%)$ & $0.004^{\star c}$ & $24(7.6 \%)$ & $0.23^{c}$ \\
\hline $\begin{array}{l}\text { History of difficulty } \\
\text { airway }\end{array}$ & $39(23.2 \%)$ & $20(5.4 \%)$ & $\begin{array}{l}< \\
0.001 * c\end{array}$ & $40(12.7 \%)$ & $0.003^{* c}$ \\
\hline Mallampati Class - I & $22(13.7 \%)$ & $51(15.3 \%)$ & $0.016^{c}$ & $22(7.7 \%)$ & $\begin{array}{l}< \\
0.001 * \mathrm{c}\end{array}$ \\
\hline II & $56(34.8 \%)$ & $152(45.5 \%)$ & - & $52(18.2 \%)$ & - \\
\hline III & $58(36 \%)$ & $76(22.8 \%)$ & - & $80(28.1 \%)$ & - \\
\hline IV & $25(15.5 \%)$ & $55(16.5 \%)$ & - & $131(46 \%)$ & - \\
\hline
\end{tabular}

BMI $\geq 35=16 / 25 / 12 / 7$, Mallampati Class $=7 / 33 / 29 / 0$. 
${ }^{\mathrm{c}}$ Chi-squared test, ${ }^{\mathrm{f}}$ Fisher's exact test.

*indicates statistical significance.

Table 1

a. Patient characteristics stratified by airway technique; combined technique as reference group.

\begin{tabular}{|c|c|c|c|c|c|}
\hline Variable & $\begin{array}{l}\mathrm{VL}+\mathrm{FOB}(\mathrm{N}= \\
66)\end{array}$ & $\begin{array}{l}\text { FOS asleep }(\mathrm{N}= \\
367)\end{array}$ & P-value & $\begin{array}{l}\text { FOS awake }(\mathrm{N}= \\
314)\end{array}$ & P-value \\
\hline $\mathrm{BMI} \geq 35$ & $18(32.7 \%)$ & $46(13.5 \%)$ & $\begin{array}{l}< \\
0.001 * c\end{array}$ & $84(27.8 \%)$ & $0.46^{c}$ \\
\hline Small mouth opening & $6(9.1 \%)$ & $20(5.4 \%)$ & $0.26^{f}$ & $24(7.6 \%)$ & $0.69^{c}$ \\
\hline Limited neck mobility & $24(36.4 \%)$ & $144(39.2 \%)$ & $0.66^{c}$ & $183(58.3 \%)$ & $0.001 * c$ \\
\hline Large neck circumference & $2(3 \%)$ & $11(3 \%)$ & $1.00^{f}$ & $9(2.9 \%)$ & $1.00^{f}$ \\
\hline $\begin{array}{l}\text { Cervical spine } \\
\text { precautions }\end{array}$ & $6(9.1 \%)$ & $47(12.8 \%)$ & $0.40^{c}$ & $24(7.6 \%)$ & $0.69^{c}$ \\
\hline $\begin{array}{l}\text { History of difficulty } \\
\text { airway }\end{array}$ & $6(9.1 \%)$ & $20(5.4 \%)$ & $0.26^{f}$ & $40(12.7 \%)$ & $0.41^{c}$ \\
\hline Mallampati Class - I & $3(4.9 \%)$ & $51(15.3 \%)$ & $\begin{array}{l}< \\
0.001 * c\end{array}$ & $22(7.7 \%)$ & $0.030^{f}$ \\
\hline$\|$ & $16(26.2 \%)$ & $152(45.5 \%)$ & - & $52(18.2 \%)$ & - \\
\hline III & $25(41 \%)$ & $76(22.8 \%)$ & - & $80(28.1 \%)$ & - \\
\hline IV & $17(27.9 \%)$ & $55(16.5 \%)$ & - & $131(46 \%)$ & - \\
\hline
\end{tabular}

${ }^{\mathrm{c}}$ Chi-squared test, ${ }^{\mathrm{f}}$ Fisher's exact test.

*indicates statistical significance. 
Table 1

b. Patient characteristics stratified by airway technique; $V L+F O B$ as reference group.

\begin{tabular}{|c|c|c|c|c|c|c|c|}
\hline Variable & $\begin{array}{l}\mathrm{VL}+\mathrm{TCl}(\mathrm{N} \\
=102)\end{array}$ & $\begin{array}{l}\mathrm{VL}+\mathrm{FOB} \\
(\mathrm{N}=66)\end{array}$ & P-value & $\begin{array}{l}\text { FOS asleep } \\
(\mathrm{N}=367)\end{array}$ & P-value & $\begin{array}{l}\text { FOS awake } \\
(\mathrm{N}=314)\end{array}$ & P-value \\
\hline $\mathrm{BMI} \geq 35$ & 23 (23.7\%) & 18 (32.7\%) & $0.23^{\mathrm{c}}$ & 46 (13.5\%) & $0.014^{c}$ & $84(27.8 \%)$ & $0.43^{\mathrm{c}}$ \\
\hline $\begin{array}{l}\text { Small mouth } \\
\text { opening }\end{array}$ & $5(4.9 \%)$ & $6(9.1 \%)$ & $0.34^{f}$ & $20(5.4 \%)$ & $0.83^{c}$ & $24(7.6 \%)$ & $0.34^{c}$ \\
\hline $\begin{array}{l}\text { Limited neck } \\
\text { mobility }\end{array}$ & 31 (30.4\%) & 24 (36.4\%) & $0.42^{c}$ & $144(39.2 \%)$ & $0.10^{c}$ & $183(58.3 \%)$ & $\begin{array}{l}< \\
0.001 * c\end{array}$ \\
\hline $\begin{array}{l}\text { Large neck } \\
\text { circumference }\end{array}$ & $8(7.8 \%)$ & $2(3 \%)$ & $0.32^{f}$ & $11(3 \%)$ & $0.043^{f}$ & $9(2.9 \%)$ & $0.041^{f}$ \\
\hline $\begin{array}{l}\text { Cervical spine } \\
\text { precautions }\end{array}$ & $2(2 \%)$ & $6(9.1 \%)$ & $0.06^{f}$ & $47(12.8 \%)$ & $0.002^{\star c}$ & $24(7.6 \%)$ & $0.039^{c}$ \\
\hline $\begin{array}{l}\text { History of } \\
\text { difficulty airway }\end{array}$ & 33 (32.4\%) & $6(9.1 \%)$ & $\begin{array}{l}< \\
0.001 * c\end{array}$ & $20(5.4 \%)$ & $\begin{array}{l}< \\
0.001 * c\end{array}$ & 40 (12.7\%) & $\begin{array}{l}< \\
0.001 * c\end{array}$ \\
\hline $\begin{array}{l}\text { Mallampati Class } \\
-I\end{array}$ & 19 (19\%) & $3(4.9 \%)$ & $\begin{array}{l}< \\
0.001 * \mathrm{c}\end{array}$ & $51(15.3 \%)$ & $0.042^{\mathrm{C}}$ & $22(7.7 \%)$ & $\begin{array}{l}< \\
0.001 * c\end{array}$ \\
\hline II & $40(40 \%)$ & $16(26.2 \%)$ & - & $152(45.5 \%)$ & - & $52(18.2 \%)$ & - \\
\hline III & 33 (33\%) & $25(41 \%)$ & - & 76 (22.8\%) & - & $80(28.1 \%)$ & - \\
\hline IV & $8(8 \%)$ & 17 (27.9\%) & - & $55(16.5 \%)$ & - & $131(46 \%)$ & - \\
\hline
\end{tabular}

${ }^{\mathrm{c}}$ Chi-squared test, ${ }^{\mathrm{f}}$ Fisher's exact test.

* indicates statistical significance.

Table 1c. Patient characteristics stratified by airway technique; $\mathrm{VL}+\mathrm{TCl}^{\mathrm{TM}}$ as reference group.

Results of the comparison of combined techniques as a group ( $\mathrm{VL}+\mathrm{FOB}$ and $\mathrm{VL}+\mathrm{TCl})$ vs FOB awake and FOB asleep are presented in Table $2 \mathrm{a}$. 


\begin{tabular}{|c|c|c|c|c|c|}
\hline Variable & $\begin{array}{l}\text { Combined }(\mathrm{N}= \\
168)\end{array}$ & $\begin{array}{l}\text { FOS asleep }(\mathrm{N}= \\
367)\end{array}$ & P-value & $\begin{array}{l}\text { FOS awake }(\mathrm{N} \\
=314)\end{array}$ & P-value \\
\hline \#Attempts - 1 & 149 (88.7\%) & 296 (80.7\%) & $0.06^{\mathrm{C}}$ & $233(74.2 \%)$ & $<.001^{\star c}$ \\
\hline 2 & $16(9.5 \%)$ & $54(14.7 \%)$ & - & $52(16.6 \%)$ & - \\
\hline$\geq 3$ & $3(1.8 \%)$ & $17(4.6 \%)$ & ${ }^{\wedge} 0.11^{\mathrm{c}}$ & $29(9.2 \%)$ & ${ }^{\wedge} 0.002^{\star c}$ \\
\hline $\begin{array}{l}\text { Minutes from in room to intubated - } \\
\text { Mean (SD) }\end{array}$ & $13.0(7.3)$ & $15.1(7.5)$ & - & $21.2(11.2)$ & - \\
\hline Median (IQR) & $12.0(9.0,15.0)$ & $13.0(10.0,18.0)$ & $0.002 * w$ & $\begin{array}{l}19.0(14.0 \\
25.0)\end{array}$ & $\begin{array}{l}< \\
0.001 * w\end{array}$ \\
\hline Range & $(0.0,65.0)$ & $(4.0,44.0)$ & - & $(6.0,96.0)$ & - \\
\hline Difficulty - Easy & $121(72 \%)$ & 231 (62.9\%) & $0.12^{c}$ & $120(38.2 \%)$ & $\begin{array}{l}< \\
0.001 * c\end{array}$ \\
\hline Difficult & $11(6.5 \%)$ & $29(7.9 \%)$ & - & 49 (15.6\%) & - \\
\hline Not rated & $36(21.4 \%)$ & $107(29.2 \%)$ & - & $145(46.2 \%)$ & - \\
\hline Traumatic - Atraumatic & $153(91.1 \%)$ & $328(89.4 \%)$ & $0.91^{f}$ & $238(75.8 \%)$ & $<.001 * f$ \\
\hline Traumatic & $1(0.6 \%)$ & $4(1.1 \%)$ & - & $3(1 \%)$ & - \\
\hline Unknown & $14(8.3 \%)$ & 35 (9.5\%) & - & $73(23.2 \%)$ & - \\
\hline
\end{tabular}

${ }^{\mathrm{c}}$ Chi-squared test, ${ }^{\mathrm{w}}$ Wilcoxon rank sum test, ${ }^{\mathrm{f}}$ Fisher's exact test.

*indicates statistical significance.

${ }^{\wedge}$ Comparing \#Attempts $>=3$ vs. $<3$.

Table 2a. Outcomes stratified by airway technique; combined technique as reference group.

Results of the combined techniques subgroup VL + FOB vs FOB awake and FOB asleep are presented in table $2 \mathrm{~b}$. 


\begin{tabular}{|c|c|c|c|c|c|}
\hline Variable & $\begin{array}{l}\mathrm{VL}+\mathrm{FOB}(\mathrm{N}= \\
66)\end{array}$ & $\begin{array}{l}\text { FOS asleep }(\mathrm{N}= \\
367)\end{array}$ & $\begin{array}{l}\mathrm{P}- \\
\text { value }\end{array}$ & $\begin{array}{l}\text { FOS awake }(\mathrm{N}= \\
\text { 314) }\end{array}$ & P-value \\
\hline \#Attempts - 1 & $57(86.4 \%)$ & $296(80.7 \%)$ & $0.65^{f}$ & $233(74.2 \%)$ & $.09^{\mathrm{c}}$ \\
\hline 2 & $7(10.6 \%)$ & $54(14.7 \%)$ & - & $52(16.6 \%)$ & - \\
\hline$\geq 3$ & $2(3 \%)$ & $17(4.6 \%)$ & ${ }^{\wedge} 0.75^{f}$ & $29(9.2 \%)$ & ${ }^{\wedge} 0.09^{c}$ \\
\hline $\begin{array}{l}\text { Minutes from in room to intubated - } \\
\text { Mean (SD) }\end{array}$ & $14.3(9.9)$ & $15.1(7.5)$ & - & $21.2(11.2)$ & - \\
\hline Median (IQR) & $\begin{array}{l}13.0(9.2 \\
17.0)\end{array}$ & $13.0(10.0,18.0)$ & $0.40^{w}$ & $19.0(14.0,25.0)$ & $\begin{array}{l}< \\
0.001 * \mathrm{w}\end{array}$ \\
\hline Range & $(0.0,65.0)$ & $(4.0,44.0)$ & - & $(6.0,96.0)$ & - \\
\hline Difficulty - Difficult & $8(12.1 \%)$ & $29(7.9 \%)$ & $0.34^{\mathrm{c}}$ & $49(15.6 \%)$ & $0.049^{c}$ \\
\hline Easy & $36(54.5 \%)$ & $231(62.9 \%)$ & - & $120(38.2 \%)$ & - \\
\hline Not rated & 22 (33.3\%) & 107 (29.2\%) & - & $145(46.2 \%)$ & - \\
\hline Traumatic - Atraumatic & $55(83.3 \%)$ & $328(89.4 \%)$ & $0.30^{f}$ & $238(75.8 \%)$ & $0.26^{f}$ \\
\hline Traumatic & $1(1.5 \%)$ & $4(1.1 \%)$ & - & $3(1 \%)$ & - \\
\hline Unknown & $10(15.2 \%)$ & 35 (9.5\%) & - & $73(23.2 \%)$ & - \\
\hline
\end{tabular}

${ }^{\mathrm{c}}$ Chi-squared test, ${ }^{\mathrm{w}}$ Wilcoxon rank sum test, ${ }^{\mathrm{f}}$ Fisher's exact test.

*indicates statistical significance.

${ }^{\wedge}$ Comparing \#Attempts > = 3 vs. $<3$.

Table 2b. Outcomes stratified by airway technique; VL + FOB as reference group.

Results of the combined techniques subgroup of $\mathrm{VL}+\mathrm{TCl}$ vs $\mathrm{VL}+\mathrm{FOB}$, FOB awake, and FOB asleep are presented in table 2c. 


\begin{tabular}{|c|c|c|c|c|c|c|c|}
\hline Variable & $\begin{array}{l}\mathrm{VL}+\mathrm{TCl} \\
(\mathrm{N}=102)\end{array}$ & $\begin{array}{l}\mathrm{VL}+\mathrm{FOB} \\
(\mathrm{N}=66)\end{array}$ & $\begin{array}{l}\mathrm{P} \text { - } \\
\text { value }\end{array}$ & $\begin{array}{l}\text { FOS asleep } \\
(\mathrm{N}=367)\end{array}$ & P-value & $\begin{array}{l}\text { FOS } \\
\text { awake }(\mathrm{N} \\
=314)\end{array}$ & P-value \\
\hline \#Attempts - 1 & $\begin{array}{l}92 \\
(90.2 \%)\end{array}$ & $\begin{array}{l}57 \\
(86.4 \%)\end{array}$ & $0.63^{f}$ & $\begin{array}{l}296 \\
(80.7 \%)\end{array}$ & $0.06^{f}$ & $\begin{array}{l}233 \\
(74.2 \%)\end{array}$ & $0.002 * c$ \\
\hline 2 & $9(8.8 \%)$ & $7(10.6 \%)$ & - & $54(14.7 \%)$ & - & $\begin{array}{l}52 \\
(16.6 \%)\end{array}$ & - \\
\hline$\geq 3$ & $1(1 \%)$ & $2(3 \%)$ & ${ }^{\wedge} 0.56^{f}$ & $17(4.6 \%)$ & ${ }^{\wedge} 0.14^{f}$ & $29(9.2 \%)$ & ${ }^{\wedge} 0.005^{\star c}$ \\
\hline $\begin{array}{l}\text { Minutes from in room to } \\
\text { intubated - Mean (SD) }\end{array}$ & $12.1(5.0)$ & $14.3(9.9)$ & - & $15.1(7.5)$ & - & $\begin{array}{l}21.2 \\
(11.2)\end{array}$ & - \\
\hline Median (IQR) & $\begin{array}{l}12.0(9.0 \\
14.0)\end{array}$ & $\begin{array}{l}13.0(9.2 \\
17.0)\end{array}$ & $0.12^{w}$ & $\begin{array}{l}13.0(10.0 \\
18.0)\end{array}$ & $\begin{array}{l}< \\
0.001^{* w}\end{array}$ & $\begin{array}{l}19.0 \\
(14.0 \\
25.0)\end{array}$ & $\begin{array}{l}< \\
0.001 * \mathrm{w}\end{array}$ \\
\hline Range & $(4.0,34.0)$ & $(0.0,65.0)$ & - & $(4.0,44.0)$ & - & $\begin{array}{l}(6.0 \\
96.0)\end{array}$ & - \\
\hline Difficulty - Difficult & $3(2.9 \%)$ & $\begin{array}{l}36 \\
(54.5 \%)\end{array}$ & $\begin{array}{l}<.001 *^{f} \\
0\end{array}$ & $29(7.9 \%)$ & $\begin{array}{l}< \\
0.001 * c\end{array}$ & $\begin{array}{l}49 \\
(15.6 \%)\end{array}$ & $\begin{array}{l}< \\
0.001 * c\end{array}$ \\
\hline Easy & $\begin{array}{l}85 \\
(83.3 \%)\end{array}$ & $8(12.1 \%)$ & - & $\begin{array}{l}231 \\
(62.9 \%)\end{array}$ & - & $\begin{array}{l}120 \\
(38.2 \%)\end{array}$ & - \\
\hline Not rated & $\begin{array}{l}14 \\
(13.7 \%)\end{array}$ & $\begin{array}{l}22 \\
(33.3 \%)\end{array}$ & - & $\begin{array}{l}107 \\
(29.2 \%)\end{array}$ & - & $\begin{array}{l}145 \\
(46.2 \%)\end{array}$ & - \\
\hline Traumatic - Atraumatic & $\begin{array}{l}98 \\
(96.1 \%)\end{array}$ & $\begin{array}{l}55 \\
(83.3 \%)\end{array}$ & $0.009^{f}$ & $\begin{array}{l}328 \\
(89.4 \%)\end{array}$ & $0.10^{f}$ & $\begin{array}{l}238 \\
(75.8 \%)\end{array}$ & $\begin{array}{l}< \\
0.001 * f\end{array}$ \\
\hline Traumatic & $0(0 \%)$ & $1(1.5 \%)$ & - & $4(1.1 \%)$ & - & $3(1 \%)$ & - \\
\hline Unknown & $4(3.9 \%)$ & $\begin{array}{l}10 \\
(15.2 \%)\end{array}$ & - & $35(9.5 \%)$ & - & $\begin{array}{l}73 \\
(23.2 \%)\end{array}$ & - \\
\hline
\end{tabular}

${ }^{\mathrm{c}}$ Chi-squared test, ${ }^{\mathrm{w}}$ Wilcoxon rank sum test, ${ }^{f}$ Fisher's exact test.

* indicates statistical significance.

${ }^{\wedge}$ Comparing \#Attempts $>=3$ vs. $<3$.

Table 2c. Outcomes stratified by airway technique; $\mathrm{VL}+\mathrm{TCl}$ as reference group.

Results of 'in room to intubation time' for different techniques are presented as destiny curves. See Figs. 3a, 3b, 3c, and 3d.

\section{Discussion}

Our study reveals important new information about the effectiveness of combined techniques $\left(\mathrm{VL}+\mathrm{FOB}\right.$ and $\left.\mathrm{VL}+\mathrm{TCl} \mathrm{I}^{\mathrm{T}}\right)$ compared to other advanced intubation techniques (awake FOB, and asleep FOB) when managing difficult intubations in a real-world setting.

We found significant differences in first pass success, failure of primary technique, speed, ease of use, and patient injury between the advanced airway techniques studied. 
Combined techniques $\left(\mathrm{VL}+\mathrm{FOB}\right.$ and $\left.\mathrm{VL}+\mathrm{TCl}^{\mathrm{T}}\right)$ achieved the highest first intubation success rate of $(88.7 \%)$ This was significantly higher than FOB awake $(74.2 \%, \mathrm{P}<0.001)$. and higher than FOB asleep $(80.7 \%, \mathrm{P}=0.06)$ In subgroup analysis of combined techniques, $\mathrm{VL}+\mathrm{TCl}^{\mathrm{TM}}$ had the highest rate of first attempt intubation success of all techniques studied (90.2\%). This was significantly higher than FOB awake $(74.2 \%, P=0.002)$ and higher than both $\mathrm{VL}+\mathrm{FOB}(86.4 \%$, $\mathrm{P}=0.63)$ and FOB asleep $(80.7 \%, \mathrm{P}=0.06)$.

The rate of first attempt intubation success rate found for $V L+F O B$ in this study is consistent with those found by Mazzinarri et. al. ${ }^{10}$ and Lenhardt et.al. ${ }^{13}$ This first attempt intubation success rate found for $\mathrm{VL}+\mathrm{TCl}^{\mathrm{IM}}$ is consistent with that found by Shah et.al ${ }^{11}$ in their case series of rescue intubations.

First attempt intubation success is important as multiple attempts are known to increase both major complications and mortality. A recent large muti-centered study demonstrated a significant increase in ICU mortality after just one attempt failure in the critically ill. ${ }^{20}$

In subgroup analysis of combined techniques, use of a $\mathrm{TCl}^{\mathrm{TM}}$ as a dynamic stylet had a higher rate of first pass success than when a FOB was used as a dynamic stylet. This is interesting as both $\mathrm{TCl}^{\mathrm{T}}$ and $\mathrm{FOB}$ possess flexible shafts and articulating tips which are important for dynamic tracheal access. ${ }^{10}$ One important difference is that a FOB requires both the right and left hand for operation, while the $\mathrm{TCl}^{\mathrm{TM}}$ requires only the right hand. Use of a FOB as a dynamic stylet necessitates a second operator handles the $\mathrm{VL}$ during the procedure. The $\mathrm{TCl}^{\mathrm{TM}}$ can be operated entirely with the right hand allowing a single operator to manage the intubation with $\mathrm{VL}$ with the left hand and $\mathrm{TCl}^{\mathrm{TM}}$ in the right. This enables a single operator to manage the entire intubation by themself. The need to coordinate two operators for VL $+F O B$ adds complexity and may reduce performance and effectiveness of $\mathrm{VL}+\mathrm{FOB}$ in comparison to $\mathrm{VL}+\mathrm{TCl}{ }^{\mathrm{T}}$. Further studies are needed to understand the role single vs double operator execution plays in first attempt success.

\section{Failure to intubate with the primary technique}

Combined techniques as a group had the lowest rate of "failure to intubate with the primary technique" at (1.8\%) followed by FOB asleep (4.6\% P = 0.11) and FOB awake (9.2\% P = 0.002). In subgroup analysis, the failure rate for VL + $\mathrm{TCl}^{\mathrm{TM}}$ was the lowest (1\%) of all techniques studied. The failure rate for $\mathrm{VL}+\mathrm{FOB}$ was $3 \%$. These failure rates are consistent with those found by Mazzinari et. al. ${ }^{10}$ for $\mathrm{VL}+\mathrm{FOB}$ and Shah et. al. ${ }^{11}$ for $\mathrm{VL}+\mathrm{TCl}{ }^{\mathrm{Tm}}$.

The failure rates of FOB asleep and FOB awake are notably higher than those found in the literature. This study was performed at a teaching institution which may account for a higher rate of failure despite removing advanced intubations performed for educational purposes. Our criteria for failure was: "three or more attempts using the same device" as this is a known point of risk inflection. It may be more stringent than other studies ${ }^{1}$ It is important to note that most studies of FOB do not define failure by number of attempts but simply by abandonment of the procedure. ElBoghdadly et. al. did report that $4.2 \%$ of patients required greater than 1 attempt. The duration of a single attempt is not reported. ${ }^{21}$ As FOB awake does not require the interruption of breathing, an single attempt may not be limited by desaturation but only by the ability of the operator and/or the patient to tolerate the attempt.

The overall performance and 'in room to intubated time' of combined techniques compared to awake FOB or asleep FOB is of particular interest as FOB has long been considered the "gold standard" for managing difficult intubations with reported success rates of up to $99 \%$. ${ }^{1722}$ While risk with multiple attempts is well established, risk related to aggregate time attempting to intubate with FOB awake has not been studied. One can only wonder if the $99 \%$ success rate of FOB reported in the literature comes at the cost of multiple and/or lengthy attempts. Persistence with a single technique is a known risk factor for airway disasters. ${ }^{16}$ 
"In room to intubated" time: Combined technique as a group (either VL + TCl ${ }^{\mathrm{TM}}$ or VL + FOB) was found to have a significantly shorter "in room to intubated" time (13.0 min) than either awake FOB or FOB asleep (15.1 mins and 21.2 min, $P=0.002$.) In subgroup analysis $\mathrm{VL}+\mathrm{TCl}^{\mathrm{TM}}$ was found to have a significantly shorter in room to intubated time" (12.1 $\mathrm{min}$ ) than FOB asleep $(15.1 \mathrm{~min}, \mathrm{P}<0.001)$ and FOB awake $(21.2 \mathrm{~min}, \mathrm{P}<0.001)$. $\mathrm{VL}+\mathrm{TCl}^{\mathrm{TM}}$ was found to have a shorter "in room to intubated" time (12.1 mins) than VL + FOB (14.3 mins, $\mathrm{P}=0.12)$ This is important as operating room time is an expensive and limited resource. Joseph et.al. studied 'in room to intubated' time differences in different awake and asleep intubation techniques and found significant increases in time associated with awake vs asleep: FOB ${ }^{22}$ To the best of our knowledge, 'in room to intubated times' for VL + FOB, and VL $+\mathrm{TCl}^{\mathrm{TM}}$ have not been studied. The causes of these increased times cannot be determined by our study. Possibilities include: (1) increased time due to intrinsic complexity of equipment needed to set up and execute each technique, (2) management and coordination of extra personnel in room for those techniques requiring 2 sets of educated hands, (3) aggregate time spent in active intubation attempts.

Further study of what factors account for the increased 'in room to intubated' time is important. Minutes spent setting up equipment or preparing the patient is expensive in terms of OR time but should not increase risk to the patient. Minutes spent in prolonged single attempts or multiple intubation attempts may cause harm. Complex and cumbersome intubation equipment increases the mental task load of providers as is evidenced by the increase in training numbers and continued practice to maintain complex FOB intubation skills. Increased mental task load is known to decrease performance and increase time need to complete a task, and may result in an increased risk of harm in the context of intubation. ${ }^{1823}$ FOBs are complex and known to require specialized training and skills to set up and operate. ${ }^{24} 22$ This complexity may require a second set of educated hands in order to set up and execute the procedure. The aggregate time spent in intubation attempts is unknown for any of the techniques studied however the 'in room to intubation time' was found to be significantly correlated to the number of intubation attempts made with the primary technique. (See table 3.)

\begin{tabular}{|c|c|c|c|c|}
\hline Variable & $1: N=749$ & 2: $N=132$ & $\geq 3: N=51$ & P-value \\
\hline Minutes from in room to intubated - Mean (SD) & $15(11)$ & $21(12)$ & $30(16)$ & $<0.001^{\star j}$ \\
\hline Median (IQR) & $14(10,18)$ & $19(13,24)$ & $27(20,39)$ & - \\
\hline Range & $(1,211)$ & $(0,84)$ & $(8,96)$ & - \\
\hline
\end{tabular}

j Jonckheere-Terpstra test.

*indicates statistical significance.

Table 3. Minutes from in room to intubated by number of attempts.

Multiple attempts take time, it seems reasonable to assume that this accounts for a portion of the 'in room to intubated' time differences observed in our study.

Operator reported ease of intubation. Combined techniques were rated as 'easy' significantly more often (72\%) than FOB awake $(38.2 \%, \mathrm{P}<0.001)$ and more often than FOB asleep $(62.9 \%, \mathrm{P}=0.12)$. In subgroup analysis, $\mathrm{VL}+\mathrm{TCl}^{\mathrm{TM}}$ was rated as 'easy' significantly more often $(83.3 \%, \mathrm{P}<0.001)$ than all other techniques studied. This is important as "difficult" implies an increased mental task load during the procedure which can lead to decreased operator performance and may lead to an increased risk of harm.

Operator reported trauma. Combined techniques as a group were reported as "atraumatic" more often (91.1\%) than either FOB asleep $(89.4 \%, \mathrm{P}=0.91)$ or FOB awake $(75.8 \%, \mathrm{P}<0.001) . \mathrm{VL}+\mathrm{TCl}^{\mathrm{TM}}$ was rated as "atraumatic "at a higher rate $(96.1 \%$ ) $)$ than any other technique studied; VL + FOB (83.3\%, P<0.009), FOB asleep $(89.4 \%, P=0.10)$, and FOB awake 
(75.8\%, $\mathrm{P}<0.001)$. These differences are interesting in that all techniques essentially use a dynamically controllable stylet for tracheal access. Multiple intubation attempts are known to increase the risk of patient harm.

We speculate that the higher first pass rates and lower failure rates found within the two combined techniques may be the result of effectively separating the role of visualization and the role of tracheal access into two separate devices: VL for visualization and a dynamic stylet for precision tracheal access. If we focus of the descriptions of the role of FOB in combined technique in the literature, over time one can see less interest in the FOB's visualization capabilities and more interest in its' navigational capabilities. The first reported combined technique series published by Sgalambro et.al. ${ }^{3}$ described using DL in combination with a FOB. The role of DL was simply as a blade to clear the airway for the passage of a FOB which provided both visualization and precision tracheal access combined in one device. The DL had little or no role in visualization. Lenhardt et.al.'s ${ }^{13}$ study combined VL and FOB using VL for visualization and FOB for both navigation and visualization. In Mazzinari et.al. ${ }^{10}$ study, the role of the FOB had atrophied to simply to that of a dynamic stylet without the use of its optical capability. Shah et.al. ${ }^{11}$ published a series in which VL was combined with the $\mathrm{TCI}^{\mathrm{TM}}$, a purpose-built articulating introducer with no intrinsic visual capabilities. With the introduction of the VL for visualization and diminishing importance of a dynamic stylet's optical capabilities, reported first-pass success in anticipated difficult intubations has trended upward. Lenhardt et.al. ${ }^{13}$ reported an $87 \%$ first attempt success rate (VL and FOB visualization), Mazzinari et.al. ${ }^{10} 91 \%$ (VL only visualization, FOB simply a dynamic stylet), and Shah et.al. ${ }^{10} 94 \%$ (VL only visualization, $\mathrm{TCl}^{\mathrm{TM}}$ purpose built dynamic stylet). This is an observation only. These studies were not designed for comparability. Studies designed to test this hypostasis properly would be of interest.

In our study, combined techniques as a group outperformed both FOB awake and FOB asleep in all endpoints measured. In subgroup analysis $\mathrm{VL}+\mathrm{TCl}^{\mathrm{TM}}$ outperformed all other techniques studied. The main limitations to widespread dissemination of combined technique are; cost, immediate availability and the need for two operators for execution. ${ }^{24} 10$ All of these drawbacks are associated with the FOB which is being cross-purposed as a dynamic stylet. In many cases, the FOB optical capabilities are not utilized, and that very feature may negatively affect the effectiveness of the combined technique in terms of ease of use, and cost. Further study is needed to understand this point

The $\mathrm{TCl}^{\mathrm{T}} \mathrm{M}$ is a novel and innovative articulating introducer without intrinsic optical capabilities. The capability and effectiveness of the $\mathrm{TCl}^{\mathrm{TM}}$ in combination with $\mathrm{VL}$ found in this study supports the need for further investigation to gain a greater understanding of the capabilities and effectiveness of different combined techniques, such as VL + FOB and VL + $\mathrm{TCl}^{\mathrm{TM}}$, in a wider variety of settings.

\section{Limitations}

This study has several important limitations mainly related to the single-center, retrospective design. First, this study is not controlled for technique selection bias, provider experience with a technique, availability of equipment, patient specific physiology, or patient anatomical features that might influence technique choice and outcomes. Second, this is a single-center study and caution must be exercised in generalizing these results widely. Third, Table 1 demonstrates significant differences in difficult intubation risk factors between groups. These differences might influence the performance or different techniques in our study. Fourth, this study includes only operating room intubations performed by anesthesia providers. Performance in other environments may vary.

Despite these limitations we believe this data provides important new insights into the performance of different advanced intubation techniques in the natural workflow of a large teaching hospital's surgical system.

Further studies, in diverse settings are needed to understand if the superior performance of combined techniques found in this study are generalizable to other systems and settings. 


\section{Conclusion}

Combined techniques as a group ( $\mathrm{VL}+\mathrm{FOB}$ and $\left.\mathrm{VL}+\mathrm{TCl}^{\mathrm{TM}}\right)$ outperformed both FOB awake and FOB asleep in terms of effectiveness, "in room to intubated" time, ease of use, and patient injury in patients with risk factors for difficult intubation. As a sub-group of combined technique, $\mathrm{VL}+\mathrm{TCl}$ outperformed all other techniques in terms of effectiveness, ease of use, and patient injury. Combined techniques may be useful as a primary approach when managing difficult intubations.

\section{Declarations}

Ethics approval: This study was approved by the University of Utah institutional Review Board \#0096411

This study was in accordance with the ethical standards of the Declaration of Helsinki (1964) and its' subsequent amendments.

As this is a retrospective chart review the University of Utah IRB waived the need for individual patient consent for this study and publication.

Consent for publication: Not Applicable

Availability of data and materials: The datasets used and/or analysed during the current study available from the corresponding author on reasonable request.

Competing interests: Sean Runnels is the inventor of the TCl Articulating introducer and hold shares in Through the Cords LLC. He was not involved in an data analysis in this study. He was involved in Study design, project management, and manuscript preparation. No other authors have declared any competing interests.

Funding: No funding was received for this study.

Authors' Contributions: K. Durnford Was responsible for database sorting and set up.

C Jacobsen was responsible for all EMR queries, database set up and management and study design.

A Shah and L. Knecht were responsible for data base evaluation, sub-group identification, and sorting.

A Presson and $\mathrm{C}$ Zhang were responsible for all statistical evaluation including preparation the statistical methods and preparation of results tables. 1 a-c, and 2 a-c and figures 3a-d.

S Runnels was responsible study design, team management and prepared figure 1 and figure 2.

A Shah, L Knecht, and S. Runnels collaborated on all portions of manuscript writing and preparation.

All authors have reviewed this manuscript.

Acknowledgments: Not applicable.

\section{References}

1. Mort TC. Emergency Tracheal Intubation: Complications Associated with Repeated Laryngoscopic Attempts.

Published online 2004:607-613. doi:10.1213/01.ANE.0000122825.04923.15 
2. Sakles JC, Chiu S, Mosier J, Walker C, Stolz U. The Importance of First Pass Success When Emergency Department. doi:10.1111/acem.12055

3. Sgalambro F, Group S, Maiolino L. An algorithm for easy intubation . Combined use of the Maclntosh laryngoscope and flexible bronchoscope in unexpected difficult intubation. 2013;(January).

4. Edwards MJ, Hutton NJ, Challis VJ, Irwin MG, Sleigh JW. Changes in airway configuration with different head and neck positions using magnetic resonance imaging of normal airways: a new concept with possible clinical applications. 2010;105(September):683-690. doi:10.1093/bja/aeq239

5. Greenland KB, Edwards MJ, Hutton NJ, Challis VJ, Irwin MG, Sleigh JW. Changes in airway configuration with different head and neck positions using magnetic resonance imaging of normal airways: a new concept with possible clinical applications. Br J Anaesth. 2010;105(5):683-690. doi:10.1093/bja/aeq239

6. Schechtman SA, Mathis M, Muller G, et al. A retrospective analysis of factors associated with difficult endotracheal tube passage with use of the hyper-angled GlideScope blade. J Head Neck Anesth. 2019;3(2). https://journals.Iww.com/johnajournal/Fulltext/2019/05000/A_retrospective_analysis_of_factors_associated.1.aspx

7. Kleine-Brueggeney M, Greif R, Schoettker P, Savoldelli GL, Nabecker S, Theiler LG. Evaluation of six videolaryngoscopes in 720 patients with a simulated difficult airway: A multicentre randomized controlled trial. $\mathrm{Br} \mathrm{J}$ Anaesth. 2016;116(5):670-679. doi:10.1093/bja/aew058

8. Bhanabhai L-R, Cormack JR, Langley B. Use of a Flexible-Tipped "Bougie" During Videolaryngoscopic Intubation in a Patient With a Base of Tongue Tumor: A Case Report. A\&A Pract. 2019;13(3). https://journals.Iww.com/aacr/Fulltext/2019/08010/Use_of_a_Flexible_Tipped_Bougie_During.4.aspx

9. Pollard J, Runnels S, Warrick C. First Case Report of Intubation With a Total Control Introducer and a Hyperangulated Video Laryngoscope. A\&A Pract. 2020;14(11):e01310. https://journals.Iww.com/aacr/Fulltext/2020/09000/First_Case_Report_of_Intubation_With_a_Total.14.aspx

10. Mazzinari G, Rovira L, Henao L, et al. Effect of Dynamic Versus Stylet-Guided Intubation on First-Attempt Success in Difficult Airways Undergoing Glidescope Laryngoscopy: A Randomized Controlled Trial. Anesth Analg. 2019;128(6):1264-1271. doi:10.1213/ANE.0000000000004102

11. Shah A. A Consecutive Case Series of Rescue Intubations with the TCI TM Articulating Introducer for Precision Tracheal Access. A\&A Pract. 2021; In Press.

12. Lenhardt R, Burkhart MT, Brock GN, Kanchi-Kandadai S, Sharma R, Akça O. Is video laryngoscope-assisted flexible tracheoscope intubation feasible for patients with predicted difficult airway? A prospective, randomized clinical trial. Anesth Analg. 2014;118(6):1259-1265. doi:10.1213/ANE.0000000000000220

13. Lenhardt R, Burkhart MT, Brock GN, Kanchi-kandadai S, Sharma R, Akça O. Is Video Laryngoscope-Assisted Flexible Tracheoscope Intubation Feasible for Patients with Predicted Difficult Airway? A Prospective, Randomized Clinical Trial. 2014;118(6):1259-1265. doi:10.1213/ANE.0000000000000220

14. Sgalambro F. The ageing brain Unexpected difficult intubation: many algorithms, many devices, many techniques , the best choice would be not having to choose . Is it utopian? Published online 2014:672-674. doi:10.1093/bja/aew349

15. Mazzinari G, Rovira L, Henao L. Effect of Dynamic Versus Stylet-Guided Intubation on First-Attempt Success in Difficult Airways Undergoing Glidescope Laryngoscopy: A Randomized Controlled Trial. 2019;128(6):1264-1271. doi:10.1213/ANE.0000000000004102

16. Cook TM, Woodall N, Frerk C. Major complications of airway management in the UK: results of the Fourth National Audit Project of the Royal College of Anaesthetists and the Difficult Airway Society. Part 1: Anaesthesia ${ }^{\& \# \times 2020 ; B r ~ J ~}$ Anaesth. 2011;106(5):617-631. doi:10.1093/bja/aer058 
17. Alhomary M, Ramadan E, Curran E, Walsh SR. Videolaryngoscopy vs. fibreoptic bronchoscopy for awake tracheal intubation: a systematic review and meta-analysis. Anaesthesia. 2018;73(9):1151-1161. doi:10.1111/anae.14299

18. Runnels S, Ferranti D, Davis AN, Pollard J. The Utah model: mental bandwidth and strategic risk generation in COVID19 airway management. Anaesthesia. 2020;75(7). doi:10.1111/anae.15086

19. Article R. Videolaryngoscopy vs . fi breoptic bronchoscopy for awake tracheal intubation: a systematic review and meta-analysis. Published online 2018:1151-1161. doi:10.1111/anae.14299

20. Russotto V, Myatra SN, Laffey JG, et al. Intubation Practices and Adverse Peri-intubation Events in Critically III Patients From 29 Countries. JAMA. 2021;325(12):1164-1172. doi:10.1001/jama.2021.1727

21. El-Boghdadly K, Onwochei DN, Cuddihy J, Ahmad I. A prospective cohort study of awake fibreoptic intubation practice at a tertiary centre. Anaesthesia. 2017;72(6):694-703. doi:10.1111/anae.13844

22. Joseph TT, Ph D, Gal JS, et al. A Retrospective Study of Success, Failure, and Time Needed to Perform Awake Intubation. 2016;(1):105-114.

23. Bourne LE, Yaroush RA. Stress and Cognition: A Cognitive Psychological Perspective. 2003;(September).

24. Mouritsen JM, Ehlers L, Kovaleva J, Ahmad I. A systematic review and cost effectiveness analysis of reusable vs . single-use fl exible bronchoscopes. Published online 2020:529-540. doi:10.1111/anae.14891

\section{Figures}

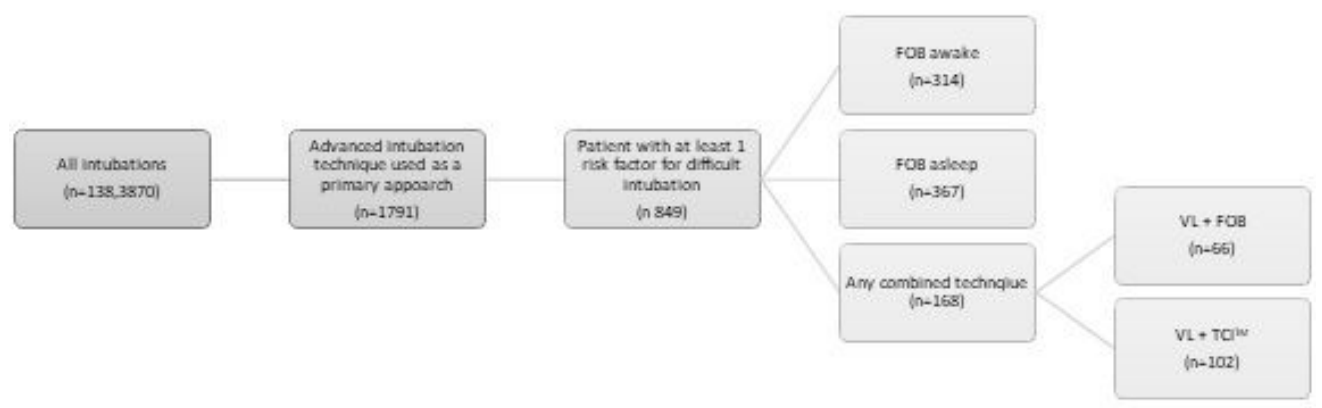

Figure 1

Data sorting flow and advanced intubation taxonomy.

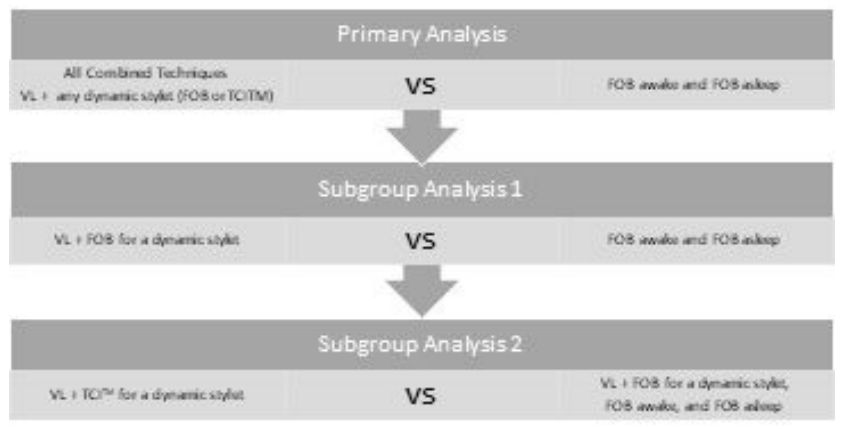

\section{Figure 2}

omparative analysis flow 

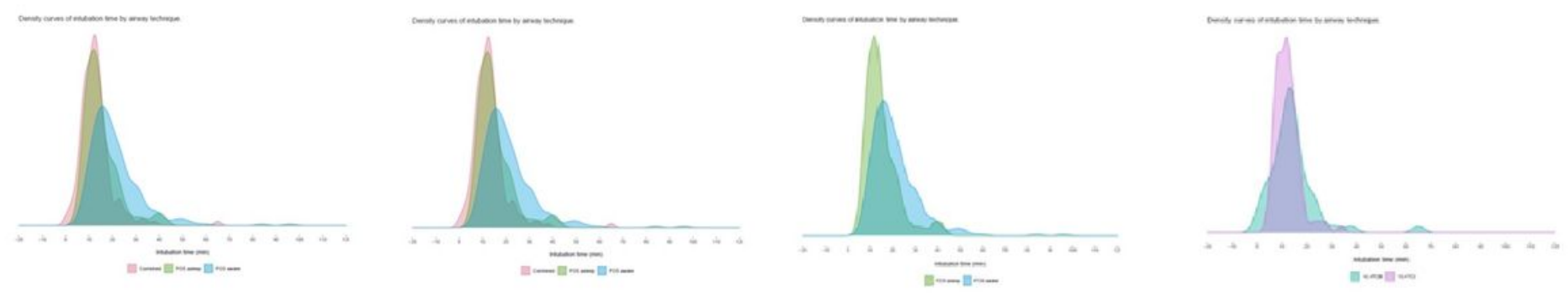

Figure 3

a. 'In room to intubated time' density curve for combined techniques as a group, FOB awake, and FOB asleep. b. 'In room to intubated time' density curve for VL + TCITM, FOB awake, and FOB asleep. c. 'In room to intubated time' density curve for FOB awake and FOB asleep. d. 'In room to intubated time' density curve for VL + FOB and VL + TCITM. 